\title{
Effects of a red card on goal-scoring in World Cup football matches
}

\author{
Jakub Červený1 ${ }^{1}$. Jan C. van Ours Or,3,5 $^{2,}$ \\ Martin A. van Tuijl ${ }^{1}$
}

Received: 13 January 2016 / Accepted: 10 April 2017 / Published online: 3 July 2017

(C) The Author(s) 2017. This article is an open access publication

\begin{abstract}
We examine the effect of the sending-off of a player on the goal-scoring rates in FIFA World Cup matches in tournaments from 1998 to 2014. We use a hazard rate framework in which the effect of a red card is modeled as a shift in the goal-scoring rate. A red card may harm the team that receives a red card and may be beneficial for their opponent. Indeed, we find that the goal-scoring rate of the sanctioned team goes down, while the goal-scoring rate of the non-sanctioned team goes up.
\end{abstract}

Keywords Association football · Goal-scoring · Red card · World Cup football matches

\section{Introduction}

A red card in association football results in the sending-off of a player. A player receives a red card after committing either a serious foul or after misconduct. A

\footnotetext{
$凶$ Jan C. van Ours

vanours@ese.eur.nl

Jakub Červený

j.cerveny@uvt.nl

Martin A. van Tuijl

m.a.vantuijl@uvt.nl

1 Department of Economics, Tilburg University, Tilburg, The Netherlands

2 Erasmus School of Economics, Erasmus University Rotterdam, Rotterdam, The Netherlands

3 Tinbergen Institute, Rotterdam, The Netherlands

4 Department of Economics, University of Melbourne, Parkville, Australia

5 CEPR, London, UK
} 
player may receive a yellow card as a caution. Receiving a second yellow card also immediately results in a red card. The team receiving the red card faces a significant disadvantage, since the number of players of such a team is reduced from eleven to ten. At the same time, the resulting power-play potentially presents a goal-scoring advantage for the opponents. However, it is also conceivable that the sending-off of a player leads to a psychological "ten do it better" effect resulting in an enhanced spirit and a better defense of the sanctioned team. This would be in line with social impact theory according to which an increase in group size decreases the perceived pressure by members to put in effort. Consequently, the sending-off of a teammate motivates the remaining players to put in more effort.

According to Anderson and Sally (2013), the strength of a team crucially depends on the weakest player. Moreover, weak players are more likely to incur a red card than more talented players. Previous research on the effects of a red card shows mixed results. This research uses various estimation methods, focusing mainly on winning or losing or on goal-scoring. The timing of a red card sanction appears to be important. For example, Ridder et al. (1994) examine 340 matches from the two Dutch professional divisions from 1991 to 1992, focusing on the scoring intensity before and after a red card. They find that the goal-scoring intensity of the team playing with 11 players increases by $88 \%$, while the goal-scoring intensity of the team with 10 players does not change. Furthermore, using the estimated results, they conclude that a red card occurring early in the match increases the odds of winning substantially, whereas the probability of victory for the sanctioned team decreases even more. They also find that around the 70th minute of a match, it becomes optimal for the defending team to commit a red card type of foul to an opposing player with a clear way to the goal.

Caliendo and Radic (2006) study FIFA World Cup matches between 1930 and 2002. Following the modeling approach of Ridder et al. (1994), they also find that the earlier a sending-off occurs in a match, the higher the odds of winning for the non-sanctioned team are. The turning point of this effect occurs around half-time, with sending-offs in the second half of a match having no effect. They do not find any supporting evidence for a "ten do it better" effect. Bar-Eli et al. (2006) study a sample of 743 games from the highest German league from 1963 to 2004. They argue that a red card is an indicator of a psychological performance crisis. Results show that when the first red card was issued, the score was more likely to be in favor of the opposing, unsanctioned team than in favor of the sanctioned team. Furthermore, a player's sending-off weakened the sanctioned team. Vecer et al. (2009) analyze the 2006 World Cup and 2008 Euro tournaments to estimate goal-scoring intensities from live betting odds. Such odds are updated during the match, reflecting the probability of an event such as one of the teams scoring a next goal. After a red card is given, the odds for scoring a next goal for both teams are updated to reflect the situation. They show that the goal-scoring intensity of the sanctioned team decreased, while the scoring intensity of an opposing team increased slightly. They find that goal-scoring in a match with a red card depends on the strength of a team. A sanction for the stronger team usually leads to fewer goals scored, whereas a sending-off for a weaker team usually leads to an increased number of goals. They also find that the optimal time to commit a foul depends on the score in a match. 
Mechtel et al. (2011) analyzing matches from the highest German league over the period 1999-2009 find that a red card given to the home team worsens their final score and result of the match. Contrary to this, the authors do not find such an effect for an away team. A sending-off occurring after the 70th minute leads to a better performance of the penalized guest team. Titman et al. (2015) study goals and bookings-both yellow cards and red cards - in two seasons (2009-2011) of English Premier League and Championship games. They distinguish between bookings and goal-scoring for the home team and the away team. Except for home red cards that only have a modest effect on home team's scoring rates for the three other combinations, home team red cards - away team scoring and away team red card on both home team and away team scoring, the red card has significant and substantial effects. Yellow cards do not affect goal-scoring rates.

Not only the effects of issuing a red card have been studied. Also, the determinants of a red card sanction are analyzed. Some studies examine whether there is a referee bias. A comprehensive analysis of yellow and red card sanctions in the 1996-2003 English Premier League seasons by Dawson et al. (2007) shows that weaker teams tend to be more sanctioned than favorites. They also find that the incidence of sanctions is higher in matches with evenly balanced teams and they find evidence for a home team bias. After controlling for team strength, the authors find that away teams collect more sanctions than home teams. Similar effects are found by Buraimo et al. (2010) in their analysis of matches over the period 2000-2006 from the highest German and English Leagues. Using betting data to distinguish between "underdogs" and favorites, they also find evidence for home team favoritism in both leagues. Pope and Pope (2015) find evidence for a player-referee own-nationality bias. They focus on the difference between fouls incurred and fouls committed by a player. A larger foul difference hence implies a larger number of beneficial foul calls. Using player-match data from the 2001-2013 UEFA Champions League seasons, they find that players officiated by a referee from the same country have a $10 \%$ increase in beneficial foul calls. This effect is even stronger for national team players as well as in later stages of the tournament. Furthermore, they find that experienced referees exhibit the same or even stronger nationality bias as less experienced referees.

In our paper, we build on previous research analyzing both the determinants of the issuing of a red card and the consequences of a red card on goal-scoring. We study World Cup matches, as these are characterized by the presence of top players and top referees. Furthermore, these matches are closely monitored (across the world). In these matches, home advantage usually does not play a role as the participating teams (except for one) never play a home match. Therefore, World Cup matches are well suited to study the effects of a red card on team performance. Our contribution to the literature is threefold: First, we investigate the determinants of the red card sanction rate focusing on the effect of the score during the match and the possible referee bias. Second, we use hazard rate models to determine the effects of a red card on the goal-scoring rate. Our model allows us to precisely determine the sequence and timing of events within a match, including also matches where no goals were scored. This is in contrast compared to Ridder et al. (1994) and Caliendo and Radic (2006) whose modeling approach requires the exclusion of such matches and assumes that goal-scoring intensity increases linearly during the match. We allow for a more flexible 
specification. Furthermore, our approach reflects the goal-scoring intensity estimates depending on actual goals scored without any further assumptions as in, e.g., Vecer et al. (2009), where estimated intensities rather reflect the evolution of betting odds during the match. Third, our data allow us to study whether there is any heterogeneity in the effects of a red card, for example whether it matters which type of player is sent off. We find that teams who are behind are more likely to receive a red card. We also find that a red card has a significantly positive effect on the team playing with more players. Contrary to this, a sending-off negatively affects the goal-scoring rate of the sanctioned team. Finally, we find that the only relevant heterogeneity in the effects of a red card is the time at which a red card was issued.

The remainder of the paper is organized as follows. Section 2 presents an overview of association football rules and the dataset used in our analysis. Section 3 describes the empirical model. Section 4 presents and discusses the parameter estimates. Section 5 concludes.

\section{Rules, theory and data}

\subsection{The rules and history}

In the quarter finals of the 1966 FIFA World Cup, England, the hosts, faced Argentina, one of the other favorites, in London, at Wembley. Ten minutes before half-time, Rudolf Kreitlein, the West-German referee, cautioned Antonio Rattin, the Argentinean captain, for the second time. This second caution inevitably meant an expulsion. The sending-off caused a major turmoil, partly due to language problems, but also partly due to the South-American suspicion of dubious West-European "home refereeing." Eventually, Rattin left the pitch, escorted by policemen. Moreover, Kreitlein also officially warned both Bobby Charlton, England's skillful attacking midfielder, and his elder brother Jack, a tough central defender. However, Alf Ramsey, the England manager, had to ask FIFA for confirmation, as few people were aware of these cautions. In the aftermath of this match, Ken Aston, an English ex-referee who then presided the FIFA's referee committee, decided that something had to be done. He proposed a card system for cautions (yellow) and expulsions (red). Rumor has it that this idea took shape in his mind when he stood in front of traffic lights in the British capital. FIFA introduced the card system at the 1970 World Cup in Mexico.

A player, substitute or substituted player is to be sent off in case of one of these seven offenses: serious foul play, violent conduct, spitting at an opponent or any other person, denying the opposing team a goal or an obvious goal-scoring opportunity by deliberately handling the ball (this does not apply to a goalkeeper within his own penalty area) denying an obvious goal-scoring opportunity to an opponent moving toward the player's goal by an offense punishable by a free kick or a penalty kick using offensive, insulting or abusive language and/or gestures and receiving a second caution in the same match. After a sending-off, the squad member in question (player, substitute or substituted player) must stay away from the field.

Prior to every World Cup tournament, the selected referees obtain instructions to focus on certain fouls and/or types of misconduct. In 1998, the emphasis was on the 
tackle from behind. In an early stage of this tournament, however, FIFA demanded the arbiters not to send off players too lightly. Expelling many world-class players would lower the level and, thus, the attractiveness of the tournament. Four years later, alertness regarding simulation ("flopping" or "diving") was given top priority. During the 2006 World Cup, the referees were supposed to pay special attention to elbowing, off-side and, once more, simulation. Four years later, they were supposed to simply focus on 'the rules of the game'. Prior to the 2014 World Cup, the arbiters were requested, like in 1998, not to expel players too easily. However, in case of elbowing, or any similar offense, they should still send off the culprit instantaneously.

\subsection{Theoretical background}

The effects of red cards in association football on the performance of football teams are interesting from an economist's point of view. Several theories have been developed to study the performance and the motivation of a team or group of members. The seminal work of French agricultural engineer Ringelmann (1913) shows that in a rope-pulling experiment individual effort decreases as the number of people involved increases. This phenomenon was later labeled as "social loafing."

Further work on the subject by Latané (1981) evolved into the social impact theory. This theory proposes that increasing the size of a group results in a decreased perceived pressure. As noted by Mechtel et al. (2011), translating this into an association football setting, an expulsion of a player should increase the pressure on the remaining players of the team. This in turn should increase the motivation and effort, leading to a better performance. This mechanism is sometimes referred to as the "ten do it better" myth (Caliendo and Radic 2006).

According to Steiner (1972), increasing the size of a group may potentially lead to problems in cooperation and coordination of the members. Hence, high individual skill levels do not necessarily translate into a better performance if the task requires a substantial degree of cooperation between group members. A successful performance of a football team undoubtedly requires the combined effort of all players. It is therefore conceivable that the cooperation of the remaining players on the pitch after a red card sanction increases.

Football matches with their red card rules present natural experiments to investigate the theoretical predictions. One of the performance indicators of a team is the goalscoring rate. The exogenous reduction in the size of a team resulting from a red card presents an excellent opportunity to investigate whether smaller teams perform better. For this, we study the effects of a red card on the goal-scoring rates of both the sanctioned team and the non-sanctioned team.

\subsection{Data and summary statistics}

Our dataset consists of 320 FIFA matches, from five World Cup tournaments from 1998 (France) to 2014 (Brazil). In 1998, the FIFA World Cup tournament in France hosted 32 teams for the first time in history. Ever since then, the tournament consists of 64 matches, viz. 48 matches in the group stage, eight matches in the round of 
Table 1 Tournament statistics

\begin{tabular}{|c|c|c|c|c|c|}
\hline \multirow[t]{2}{*}{ Tournament } & \multirow{2}{*}{\multicolumn{2}{|c|}{ Number }} & \multicolumn{3}{|c|}{ Minutes until first RC } \\
\hline & & & Average & Min & Max \\
\hline \multicolumn{6}{|c|}{ Panel A: Red cards } \\
\hline 1998 & \multicolumn{2}{|l|}{22} & 62 & 19 & 89 \\
\hline 2002 & \multicolumn{2}{|l|}{17} & 64 & 22 & 103 \\
\hline 2006 & \multicolumn{2}{|l|}{28} & 65 & 28 & 120 \\
\hline 2010 & \multicolumn{2}{|l|}{17} & 66 & 24 & 120 \\
\hline 2014 & \multicolumn{2}{|l|}{10} & 56 & 37 & 94 \\
\hline Total/avg. & 94 & & 63 & 19 & 120 \\
\hline \multirow[t]{2}{*}{ Tournament } & \multirow[t]{2}{*}{ Average } & \multirow[t]{2}{*}{ No RC } & \multicolumn{3}{|c|}{ Matches with RC } \\
\hline & & & Average & Before RC & After RC \\
\hline \multicolumn{6}{|c|}{ Panel B: Goals per match } \\
\hline 1998 & 2.67 & 2.58 & 2.81 & 1.25 & 1.56 \\
\hline 2002 & 2.51 & 2.62 & 2.07 & 1.07 & 1.00 \\
\hline 2006 & 2.30 & 2.34 & 2.20 & 1.45 & 0.75 \\
\hline 2010 & 2.27 & 2.36 & 2.00 & 1.12 & 0.88 \\
\hline 2014 & 2.67 & 2.74 & 2.30 & 1.20 & 1.10 \\
\hline Total/avg. & 2.48 & 2.53 & 2.30 & 1.22 & 1.06 \\
\hline
\end{tabular}

Based on 320 matches

$R C$ red card

sixteen, four quarter finals, two semifinals, a position match (for the third place) and the final. Moreover, starting from the 1998 FIFA World Cup, teams were allowed to bring on three substitutes, as compared to two players and one goalkeeper in 1994, now without any restrictions. This gives coaches ample opportunities to substitute players who have already received a yellow card, thus avoiding that these players receive a second yellow card, i.e., a red card.

Table 1 provides summary statistics of the dataset. In total, our dataset includes 94 red cards. The highest number of 28 red cards was awarded in 2006 (Germany), followed by 22 red cards in 1998. In both the 2002 and 2010 tournaments played in South Korea/Japan and South Africa, respectively, 17 red cards were awarded. Finally, the lowest number of only 10 red card sanctions occurred in 2014. As can be seen, most red cards are issued in the second half of the match, on average around the 65th minute. The only deviation is the 2014 tournament, where on average a red card was awarded in the 56th minute. The earliest red card was given in the 19th minute, and the first red card that was given in the latest stage of match was in the 120th minute in 2006 and 2010.

As shown in Panel B, the average number of goals per match has fallen from 2.67 in 1998 to 2.27 in 2010 . The 2014 tournament reversed this trend with 2.67 goals per match scored. On average, more goals were scored in matches without a red card. Also, in matches with at least one red card, fewer goals were scored after the red card 
Table 2 Sample characteristics

Based on 320 matches

$R C$ red card; player

characteristics refer to players receiving a red card. Defender includes goalkeeper

\begin{tabular}{lrll}
\hline Variable & Mean & Min & Max \\
\hline Panel A: Match characteristics & & & \\
Rank & 21.30 & 1 & 105 \\
Group stage & 0.75 & 0 & 1 \\
16 finals & 0.12 & 0 & 1 \\
Quarterfinals & 0.06 & 0 & 1 \\
Semifinals & 0.03 & 0 & 1 \\
Final/3rd place & 0.03 & 0 & 1 \\
Goal difference at RC & -0.44 & -3 & 2 \\
Goals scored & 1.24 & 0 & 8 \\
Same continent referee & 0.06 & 0 & 1 \\
Referee rank & 5.28 & 1 & 10 \\
Referee rank missing & 0.54 & 0 & 1 \\
Panel B: Player characteristics & & & \\
Age & 2.74 & 2.1 & 3.5 \\
Caps & 4.17 & 0.1 & 11.3 \\
Midfielder & 0.43 & 0 & 1 \\
Forward & 0.22 & 0 & 1 \\
Defender & 0.35 & 0 & 1 \\
Midfielder & 0.44 & 0 & 1 \\
Forward & 0.21 & 0 & 1 \\
\hline & & &
\end{tabular}

was issued than before. However, the average duration of the match after a red card is much shorter than the duration up to the first red card. Taken this difference in duration into account, it is clear that the goal-scoring intensity is higher after a red card than before.

Table 2 gives an overview of the characteristics of our sample (the definition of the variables is given in "Appendix"). The rank of the team ranges from one to 105 with an average of 21.3. The goal difference at the time the first red card was issued ranges from minus three to plus two with an average of -0.44 . Tactics of teams usually differ between group and elimination rounds of a tournament. Also, team strength is usually more equal as weak teams are already out. Therefore, in our analysis we also include a dummy variable indicating whether a match is played in the group phase or later stages of the tournament. The total number of goals scored per match ranges from 0 to 8. In the analysis, we will investigate to what extent the referee matters. For this, we use two indicators. First, whether the referee is from the same continent as the team for which we analyze the goal-scoring rate or the red card sanction rate. Second, we take the rank of the referee 1 year prior to the tournament into account. The bottom rows of Table 2 show which type of players received a first red card in the game.

Table 3 gives the distribution of the red cards by $15 \mathrm{~min}$ intervals in the match. No red card was issued in the first $15 \mathrm{~min}$ of any match. Most red cards were given in the last $15 \mathrm{~min}$ of regular time; only five red cards were issued in extra time. This means that in about $25 \%$ of the matches at least one red card was issued, while in the 
Table 3 Distribution of red cards per $15 \mathrm{~min}$ intervals
Based on 320 matches of which 27 matches with extra time. Two red cards were given to players on the bench and are thus not included in the analysis

\begin{tabular}{lccc}
\hline Minute & First & Later & Total \\
\hline $0-15$ & 0 & 0 & 0 \\
$16-30$ & 9 & 0 & 9 \\
$31-45$ & 19 & 0 & 19 \\
$46-60$ & 14 & 1 & 15 \\
$61-75$ & 16 & 1 & 17 \\
$76-90$ & 22 & 6 & 28 \\
$91-105$ & 1 & 0 & 1 \\
$106-120$ & 3 & 0 & 3 \\
Total & 84 & 8 & 92 \\
\hline
\end{tabular}

extra time matches this was less than $20 \%$. The focus of our analysis will be on the 84 first red cards, ignoring eight later red cards and two red cards that were presented to players on the bench.

\subsection{Exploratory analysis}

Before analyzing the effect of a red card on the goal-scoring rate, we present a preliminary analysis of the effect of a red card on the outcome of a match. This is a similar approach as used by Mechtel et al. (2011). We first estimate a simple probit model which takes the following form:

$$
\operatorname{Pr}\left(y_{i}=1 \mid z_{i}^{\prime}\right)=\Phi\left(z_{i}^{\prime} \xi+d_{r c} \gamma_{1}+d_{r c}^{o} \gamma_{2}\right)
$$

where $y_{i}$ is the outcome variable taking a value of 1 if the team won a match, $z_{i}^{\prime}$ is vector of explanatory variables, the parameters $\gamma_{1}$ and $\gamma_{2}$ capture the effect of an opponent's and an own red card on the outcome of a match and $\Phi$ is the cumulative standard normal distribution function. We estimate the probability of winning the match for both teams. From the total of 320 matches, this results in 640 observations used in the analysis. In addition to this, we estimate a linear model including the same explanatory variables, with a dependent variable taking values of 0 if the team loses, 1 for a draw and 2 for a win. ${ }^{1}$ The results are summarized in Table 4 . Columns (1) and (2) present estimates from the probit model and columns (3) and (4) from the linear model. Columns (2) and (4) are excluding extra time results.

It is clear that a higher number of goals scored increase the probability of winning a match substantially. Figure 1 plots the probability of winning as a function of the number of goals scored (the gray area represents the $95 \%$ confidence interval). After scoring the first goal, the probability of winning is equal to $22 \%$. Scoring a second goal increases the probability of winning to $68 \%$, whereas the third goal takes the odds of winning up to $95 \%$. Similarly, the probability of winning a match increases if the opposing team receives red card. For the team penalized with a red card sanction, the

\footnotetext{
1 We also investigate an ordered probit specification finding very similar results.
} 
Table 4 Parameter estimates probability to win a match

\begin{tabular}{|c|c|c|c|c|c|c|c|c|}
\hline \multirow[b]{3}{*}{ Opponent RC } & \multicolumn{4}{|c|}{ Probit model } & \multicolumn{4}{|c|}{ Linear model } \\
\hline & \multicolumn{2}{|l|}{ (1) } & \multicolumn{2}{|l|}{ (2) } & \multicolumn{2}{|l|}{ (3) } & \multicolumn{2}{|l|}{ (4) } \\
\hline & $0.23 * *$ & (3.3) & $0.24 * * *$ & $(3.4)$ & $0.47 * * *$ & $(5.8)$ & $0.50 * * *$ & $(6.1)$ \\
\hline Own RC & $-0.28 * * *$ & $(8.3)$ & $-0.31 * * *$ & $(9.2)$ & $-0.37 * * *$ & $(4.3)$ & $-0.41 * * *$ & $(4.7)$ \\
\hline Rank opponent & $0.06 * * *$ & $(5.1)$ & $0.06^{* * *}$ & $(5.1)$ & $0.12 * * *$ & $(7.3)$ & $0.12 * * *$ & $(7.2)$ \\
\hline Rank own & $-0.08 * * *$ & $(5.6)$ & $-0.08 * * *$ & $(5.5)$ & $-0.12 * * *$ & $(7.4)$ & $-0.12 * * *$ & $(7.2)$ \\
\hline 16 finals & -0.07 & (1.4) & 0.05 & $(1.3)$ & -0.01 & $(0.5)$ & -0.01 & $(0.4)$ \\
\hline Quarterfinals & -0.06 & $(0.9)$ & -0.03 & $(0.4)$ & $-0.03 *$ & $(1.7)$ & -0.02 & (1.6) \\
\hline Semifinals & -0.04 & $(0.6)$ & 0.01 & $(0.1)$ & -0.03 & $(1.3)$ & -0.03 & $(1.1)$ \\
\hline Final/3rd place $m$. & -0.05 & $(0.6)$ & 0.06 & $(1.1)$ & -0.05 & $(1.4)$ & -0.05 & $(1.3)$ \\
\hline Goal diff at RC & $0.21 * *$ & $(2.7)$ & $0.20 * *$ & $(2.5)$ & $0.22 * *$ & $(3.5)$ & $0.20 * *$ & $(3.2)$ \\
\hline Constant & $-0.24 * *$ & $(2.4)$ & $-0.24 * *$ & $(2.6)$ & $1.00 * * *$ & (111.7) & $1.01 * * *$ & (111.2) \\
\hline Log likelihood & 355.0 & & 360.2 & & 725.6 & & 743.9 & \\
\hline Extra time included & Yes & & No & & Yes & & No & \\
\hline
\end{tabular}

640 observations; absolute $t$ statistics in parentheses; standard errors clustered at the match level Probit model: probability of winning a match, marginal effects; linear model: 0 for a loss, 1 for a draw, 2 for a win; rank divided by 10

$R C$ red card

${ }^{*} p<0.10 ;{ }^{* *} p<0.05 ;{ }^{* * *} p<0.001$

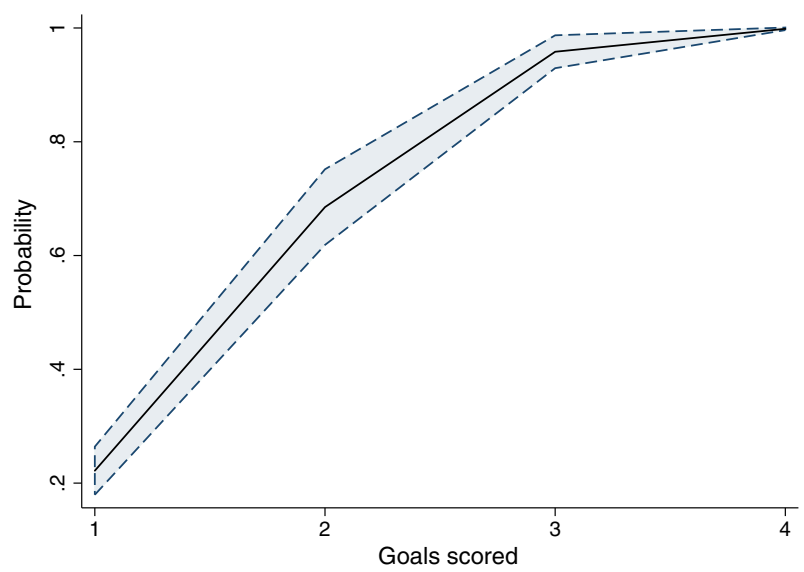

Fig. 1 Probability to win as function of goals scored.

Note The dotted lines represent the $95 \%$ confidence interval

odds of winning decrease substantially. The lower the ranking of the opponent, the higher the probability of winning a match. Conversely, the lower the ranking of the team itself, the lower the odds of winning. The stage of tournament does not seem to have any effect. The more goals a team is ahead of its opponent before the red card, the higher the probability of winning the match.

At the mean value of the explanatory variables, the marginal probability of winning a match before a red card sanction is equal to $29 \%$. After an opponent's red card 
A

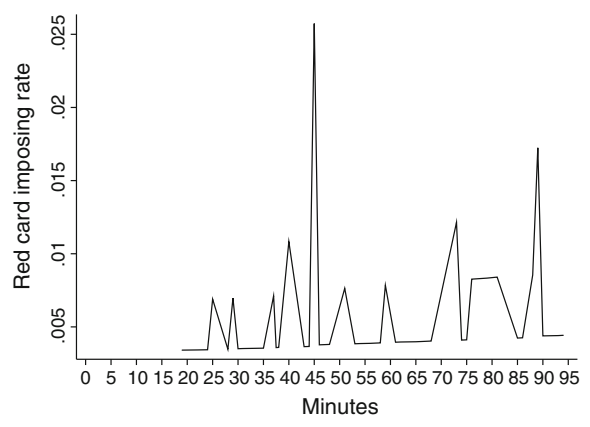

B

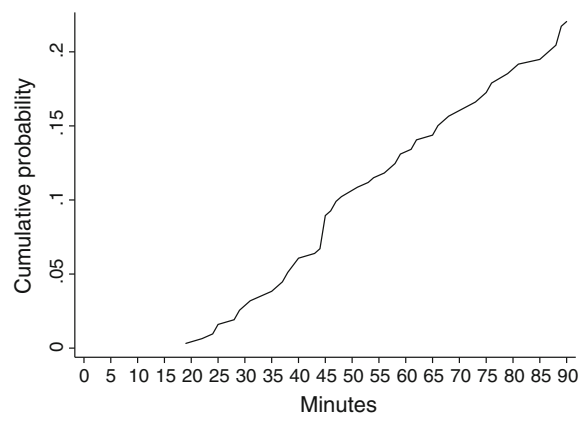

Fig. 2 Red card imposing rate and cumulative red card probability. a Red card rate. b Cumulative red card probability

sanction, the odds of winning increase by 23 percentage points to almost $52 \%$. After the team itself receives a red card, the odds of winning decrease from $35 \%$ to only $7 \%$. The results shown in columns (3) and (4) make it clear that these are not dependent on the specific functional form assumptions underlying the probit model and the linear model.

\section{Setup of the analysis}

\subsection{Imposition of a red card}

A red card foul is a phenomenon that usually occurs later in a football match but very rarely within the first $20 \mathrm{~min}$ of a match. In our dataset, the earliest red card awarded was in the 19th minute. This is shown in Fig. 2 which plots the rate at which red cards were issued in a match. The highest occurrence of red card sanctions is around the 45 th minute of a match, with another peak around the 75th minute. Figure 2 also plots the cumulative probability that a red card is imposed. Within the first half of the match, the probability of committing a red card offense is less than $10 \%$. In the course of the second half of a match, this figure rises to slightly more than $20 \%$.

We model the rate by which a red card is imposed using a mixed proportional hazard model. The red card sanction rate at time $t$ conditional on observed characteristics $x$ and unobserved characteristics $v$ is specified as follows ${ }^{2}$ :

$$
\theta_{r}(t \mid x, v)=\lambda_{r}\left(t_{i}\right) \exp \left(x^{\prime} \beta_{r}+v\right)
$$

\footnotetext{
2 The end of the first half of the match is assumed to be at the 45th minute. The end of the second half of the match is assumed to have an injury time of $5 \mathrm{~min}$ for all matches included in the analysis. The extra time matches are modeled as follows: The first part of the extra time is assumed to be $15 \mathrm{~min}$, while the second part is assumed to be $17 \mathrm{~min}$, so including $2 \mathrm{~min}$ of injury time. In some tournaments, matches in extra time could have been concluded by a golden goal. Then, match time stops at the moment a golden goal is scored.
} 
where $\beta_{r}$ is a vector of parameters. The duration dependence is flexibly modeled using step function:

$$
\lambda_{r}\left(t_{i}\right)=\exp \left(\sum_{k} \lambda_{k, r} I_{k}(t)\right)
$$

where $k(=1, \ldots, K)$ is a subscript for match-intervals and $I_{k}(t)$ are time-varying dummy variables for subsequent match-intervals. The match is divided into two halves. We estimate a constant and normalize $\lambda_{0, r}=0$. Extra time matches include one additional interval. The conditional density function for the completed durations until a red card was issued can be written as:

$$
f(t \mid x, v)=\theta_{r}(t \mid x, v) \exp \left(-\int_{0}^{t} \theta_{r}(t \mid x, v) \mathrm{d} s\right)
$$

Several unobserved factors such as coach tactics, weather or match attendance might affect the goal-scoring rate. We assume that these unobservables $v$ come from a discrete distribution $G$ with two points of support $\left(v_{1}, v_{2}\right)$, representing two types of teams, one with a low red card rate and another with high red card rate. The associated probabilities are denoted as follows: $\operatorname{Pr}\left(v=v_{1}\right)=p_{1}, \operatorname{Pr}\left(v=v_{2}-v_{1}\right)=p_{2}$, where $p_{j}(j=1,2)$ is assumed to have a logistic distribution. We remove the unobserved heterogeneity distribution through integration:

$$
f(t \mid x)=\int_{v} f(t \mid x, v) \mathrm{d} G(v)
$$

In the analysis for matches without a red card, the duration of the red card is assumed to be censored at the end of the match. The likelihood function is optimized using the method of maximum likelihood.

\subsection{The effects of a red card}

Figure 3 plots the goal-scoring rate before and after a first red card was issued, focusing on the first goal scored before the red card (this includes also matches with no red cards given) and the first goal scored after a red card sanction. The goal-scoring rate is higher after a red card has been issued. The goal-scoring rate after a team receives a red card appears to be lower compared to a situation when the opponent receives a red card. There seems to be an increase in the goal-scoring rate toward the end of a match, a well-known fact found also in previous studies (Van Ours and van Tuijl 2011).

The focus of our analysis is on the effect of a first red card on the goal-scoring rate of both the sanctioned and the non-sanctioned team. Each match is divided into several periods by the goals scored and the red cards given, with episodes from a goal to a red card or to the end of a match being right-censored. ${ }^{3}$ The effect of the red card is modeled as a shift in the goal-scoring (hazard) rate. To estimate the effect, we use

\footnotetext{
3 Only one red card in the dataset resulted in a penalty kick. Excluding this observation has no effects on the results.
} 


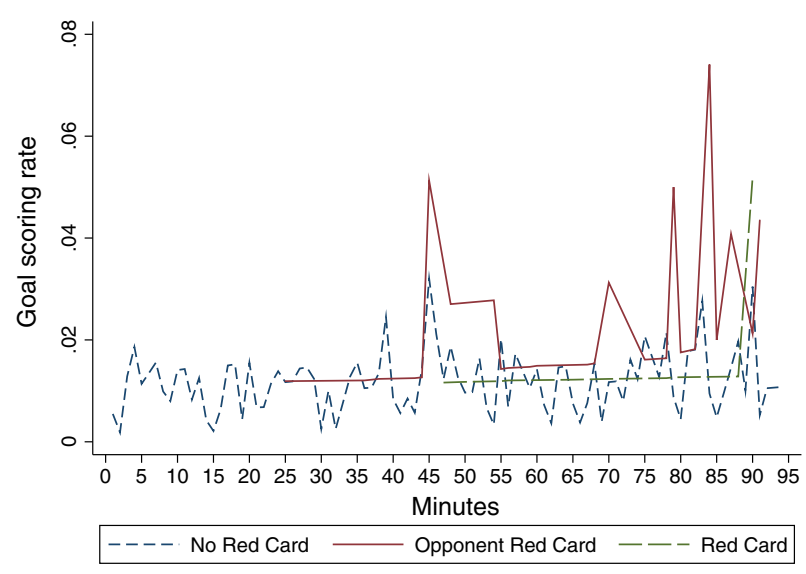

Fig. 3 Goal-scoring rate before and after red card

a mixed proportional hazard framework which allows the rate of transition from one state to the other to be affected by individual observed and unobserved characteristics as well as the duration of the stay in a state (Heckman and Singer 1984). In our case, the state is defined by the current number of goals scored by the team, considering also whether or not a red card was given. The transition rate is equivalent to the goal-scoring rate. As additional observed characteristics, we use the pre-tournament FIFA ranking of both teams and the current stage of the tournament. We also take a possible referee bias into account. Thus, the goal-scoring rate at the time of a match $t_{i}$ conditional on the observed characteristics $x$, the time of the opponent's the red card sanction $t_{r c}$, time of the own red card sanction $t_{r c}^{o}$ and the unobserved characteristics $v$, the model is specified as follows:

$$
\theta\left(t_{i} \mid x, t_{r c}, t_{r c}^{o}, v\right)=\lambda\left(t_{i}\right) \exp \left(x^{\prime} \beta+\delta_{1} I\left(t_{i}>t_{r c}\right)+\delta_{2} I\left(t_{i}>t_{r c}^{o}\right)+v\right)
$$

where $\beta$ is a vector of parameters. The parameter $\delta_{1}$ describes how the hazard rate shifts at the moment a red card was issued and thus measures the effect of an opponent's red card on the goal-scoring rate. Similarly, the parameter $\delta_{2}$ measures the shift of the goal-scoring rate after a team receives a red card. ${ }^{4}$ The subscript $i$ denotes the period from a goal scored or a red card awarded. The first period from the beginning of the match up until the first event (a goal scored or a red card issued) is denoted as $i=1$. The duration dependence is flexibly modeled using step function:

$$
\lambda\left(t_{i}\right)=\exp \left(\sum_{k} \lambda_{k} I_{k}\left(t_{i}\right)\right)
$$

\footnotetext{
4 Note that we model only one of these events. If a second red card is imposed, match time stops at which point the goal-scoring rate is assumed to be right-censored.
} 
where $k(=1, \ldots, K)$ is a subscript for match-intervals and $I_{k}(t)$ are time-varying dummy variables for subsequent match-intervals. The match is divided into two halves, assuming an injury time of $5 \mathrm{~min}$. Extra time matches include one additional interval, allowing for a third injury time of $2 \mathrm{~min} .{ }^{5}$ We estimate a constant and normalize $\lambda_{0}=0$. The conditional density function for the completed durations until goal scored can be written as:

$$
f\left(t_{i} \mid x, t_{r c}, t_{r c}^{o}, v\right)=\theta\left(t_{i} \mid x, t_{r c}, t_{r c}^{o}, v\right) \exp \left(-\int_{t_{i-1}}^{t_{i}} \theta\left(t_{i} \mid x, t_{r c}, t_{r c}^{o}, v\right) \mathrm{d} s\right)
$$

Note that $t_{i-1}$ denotes the time of a previous event in the match, either a goal scored or a red card issued. Several unobserved factors such as coach tactics, the weather or match attendance might affect the goal-scoring rate. We assume that the random effects $v$ come from a discrete distribution $G$ with two points of support $\left(v_{1}, v_{2}\right)$, representing two types of teams, one with a low goal-scoring rate and another with a high goal-scoring rate. The associated probabilities are denoted as follows: $\operatorname{Pr}(v=$ $\left.v_{1}\right)=p_{1}, \operatorname{Pr}\left(v=v_{2}-v_{1}\right)=p_{2}$, where $p_{n}(n=1,2)$ is assumed to have a logistic distribution. We remove the unobserved heterogeneity distribution through integration:

$$
f\left(t_{i} \mid x, t_{r c}, t_{r c}^{o}\right)=\int_{v} f\left(t_{i} \mid x, t_{r c}, t_{r c}^{o}, v\right) \mathrm{d} G(v)
$$

The resulting log-likelihood function equals:

$$
\mathcal{L}=\sum_{j=1}^{N} \log \left\{\prod_{i=1}^{I} \theta\left(t_{i} \mid x, t_{r c}, t_{r c}^{o}\right)^{d_{i}} \exp \left(-\int_{t_{i-1}}^{t_{i}} \theta\left(t_{i} \mid x, t_{r c}, t_{r c}^{o}\right)\right)\right\}
$$

considering a dataset consisting of $j=1, \ldots, N$ matches, $i=1, \ldots, I$ denotes the period in a match from the first goal scored or red card issued, with $I$ being the last part from a goal scored or a red card awarded until the end of match, while $d_{i}$ denotes the indicator of whether a goal was scored. The parameters of the likelihood function are estimated using the method of maximum likelihood.

\section{Parameter estimates}

\subsection{Red card sanction rate}

Table 5 presents the parameter estimates of the red card sanction rate. The FIFA ranking of teams does not seem to have an effect on the rate at which red cards are issued during a match. Compared to the group stage, later stages of a tournament also do not have an effect. As expected, the more goals ahead, the lower the probability of conducting a red card foul. With each goal ahead, the probability decreases by

\footnotetext{
5 We also test for an alternative specification of 15 -min intervals finding that our main parameter estimates are not affected by this.
} 


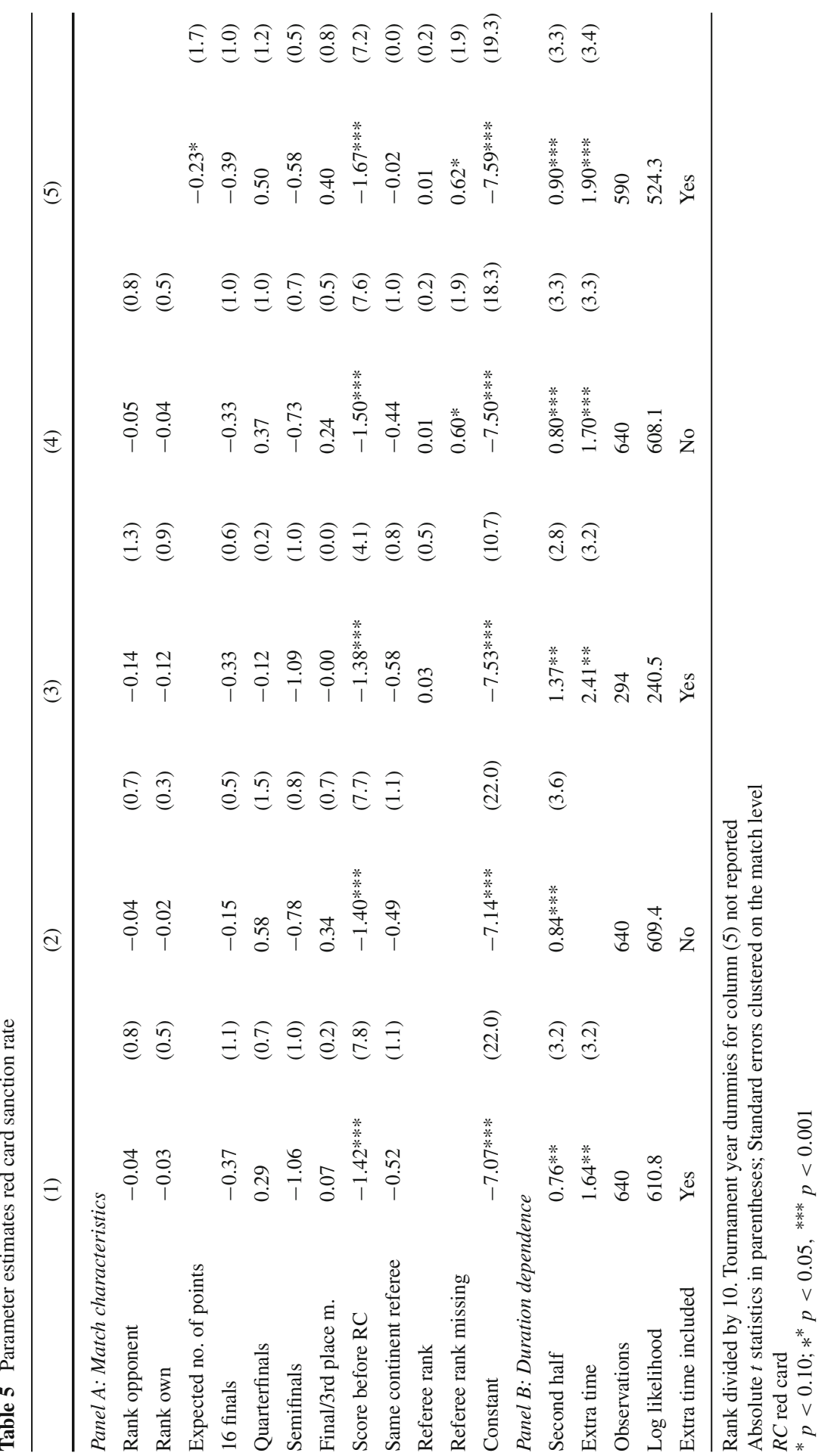


$(\exp (-1.42)-1) \times 100 \approx 75 \%$. Column $(2)$ presents estimates excluding extra time matches.

From the first two columns, it is a clear that the imposition of a red card is not related to the rank of a team or its opponent. The stage of tournament is not important either. The only determinant of the red card sanction rate is the goal difference. If a team has conceded more goals than it scored, it is more likely to receive a red card.

Furthermore, a red card is more likely to be issued in the second half of a match and in particular in extra time. In column (3), we add the rank of the referee as an additional explanatory variable. The number of observations drops substantially since there were many referees for whom no ranking is available. The ranking of the referee has no effect on the red card sanction rate. In column (4), we introduce a dummy variable for matches in which no referee ranking is available. This dummy variable has a positive effect suggesting that less skillful referees are more likely to issue a red card.

Nevertheless, the dummy variable is not different from zero at conventional levels of significance. Finally, as an alternative to the FIFA ranking, we use bookmaker data as an indication of the difference in strength between two teams in a match. We translate pre-match betting odds into an expected number of points. ${ }^{6}$ The relevant parameter estimates are presented in column (5). There is no significant effect of the expected number of points on the red card sanction rate. In an additional sensitivity analysis, we investigated whether unobserved heterogeneity is important, but we did not find evidence of this.

\subsection{The effects of a red card on the goal-scoring rate}

Table 6 shows the parameter estimates of the goal-scoring rate. Initially, we ignore potential unobserved heterogeneity in the goal-scoring rate. The first two columns of the table present the effects making a distinction between the own team's and the opponent's red card. Columns (3) and (4) extend the analysis by introducing heterogeneity in the red card effect according to the time the card was issued. Clearly, there is a highly significant positive effect on the goal-scoring rate after a red card is imposed to the opposing team. The probability of scoring a goal increases by almost $(\exp (0.81)-1) \times 100 \approx 124 \%$. In line with this, receiving a red card sanction has significantly negative effects on the goal-scoring rate, lowering the rate by $47 \%$. The estimated coefficients of the FIFA ranking variables suggest that a lower ranking of a team translates to a lower goal-scoring rate.

The higher the rank of a team, the lower the goal-scoring rate; the lower the ranking of the opponent, the higher the goal-scoring rate. The stage of a tournament does not seem to have much of an effect. The only difference being the quarterfinal stage where the goal-scoring rate is to be lower as compared to the group stage. Whether or not a referee was from the same continent does not influence the goal-scoring rate. The

\footnotetext{
6 See Van Ours and van Tuijl (2016) and the appendix for details. The number of observations decreases slightly since pre-match betting odds are not available for all matches. Because we were unable to find odds from a single source we use tournament-specific dummies to control for possible differences in the structure of betting odds between tournaments.
} 
Table 6 Parameter estimates goal-scoring rate

\section{(1)}

\begin{tabular}{|c|c|c|c|c|c|c|c|c|}
\hline \multicolumn{9}{|c|}{ Panel A: Match characteristics } \\
\hline Rank opponent & $0.10^{* * *}$ & $(5.0)$ & $0.10 * * *$ & $(5.1)$ & $0.10 * * *$ & $(4.8)$ & $0.10^{* * *}$ & $(4.9)$ \\
\hline Rank own & $-0.16^{* * *}$ & $(5.6)$ & $-0.16^{* * *}$ & $(5.8)$ & $-0.16 * * *$ & $(5.7)$ & $-0.16^{* * *}$ & $(5.8)$ \\
\hline 16 finals & -0.14 & $(1.3)$ & -0.19 & (1.5) & -0.15 & (1.4) & $-0.21^{*}$ & (1.6) \\
\hline Quarterfinals & $-0.38^{*}$ & $(1.8)$ & -0.28 & $(1.4)$ & $-0.38^{*}$ & (1.8) & -0.28 & $(1.4)$ \\
\hline Semifinals & -0.31 & $(1.0)$ & -0.31 & $(0.9)$ & -0.31 & $(1.0)$ & -0.31 & $(0.9)$ \\
\hline Final/3rd place $m$. & 0.08 & $(0.4)$ & 0.12 & $(0.6)$ & 0.05 & $(0.2)$ & 0.12 & $(0.6)$ \\
\hline Same continent ref. & 0.06 & $(0.4)$ & 0.07 & $(0.5)$ & 0.05 & $(0.3)$ & 0.06 & $(0.4)$ \\
\hline Constant & $-4.36^{* * *}$ & $(47.4)$ & $-4.36^{* * *}$ & $(47.1)$ & $-4.34 * * *$ & $(46.5)$ & $-4.34 * * *$ & $(46.2)$ \\
\hline \multicolumn{9}{|c|}{ Panel B: Duration dependence } \\
\hline Second half & $0.16 * *$ & $(2.3)$ & $0.17 * *$ & $(2.4)$ & $0.16^{* *}$ & $(2.3)$ & $0.16 * *$ & $(2.3)$ \\
\hline Extra time & 0.12 & $(0.4)$ & & & 0.11 & $(0.4)$ & & \\
\hline \multicolumn{9}{|l|}{ Panel C: Red card effects } \\
\hline Effect RC & $0.81 * * *$ & $(6.7)$ & $0.80 * * *$ & $(6.5)$ & & & & \\
\hline Effect own RC & $-0.63 * *$ & $(2.2)$ & $-0.66^{* *}$ & $(2.3)$ & & & & \\
\hline Effect RC 0-45 & & & & & $0.80^{* * *}$ & $(4.8)$ & $0.80^{* * * *}$ & $(4.9)$ \\
\hline Effect RC 45-75 & & & & & $0.53^{* *}$ & $(2.6)$ & $0.56^{* *}$ & $(2.7)$ \\
\hline Effect RC 75-120 & & & & & $1.41 * * *$ & $(6.6)$ & $1.35^{* * *}$ & $(5.8)$ \\
\hline Effect own RC 0-45 & & & & & $-1.19 * *$ & $(3.0)$ & $-1.19 * *$ & (3.0) \\
\hline Effect own RC 45-75 & & & & & -0.30 & $(0.7)$ & -0.40 & $(0.9)$ \\
\hline Effect own RC 75-120 & & & & & 0.51 & $(0.7)$ & 0.52 & $(0.7)$ \\
\hline Log likelihood & 4299.8 & & 4192.1 & & 4293.6 & & 4187.2 & \\
\hline Wald test $\chi^{2}$ & & & & & $49.3^{* * *}$ & & $40.3^{* * *}$ & \\
\hline Wald test DF & & & & & 4 & & 4 & \\
\hline
\end{tabular}

Rank divided by 10. Tournament year dummies for column (5) not reported $R C$ red card

640 observations; absolute $t$ statistics in parentheses; Standard errors clustered at the match level ${ }^{*} p<0.10 ; *^{*} p<0.05 ;{ }^{* * *} p<0.001$

goal-scoring rate is higher in the second half of a match than in the first half. In extra time, the goal-scoring rate is highest.

From columns (3) and (4), it appears that the effect of a red card given between the 45 th and 75 th minute of a match lowers the goal-scoring rate when compared to red cards given either earlier or later in a match. The parameter estimate of a red card received between the 75th and the 120th minute shows some evidence of the "ten do it better" myth. However the parameter estimate is statistically not significantly different from zero. The result of the Wald test confirms that the additional time-specific red card effects significantly improve the model fit. Apparently, the timing of the red card is important. Columns (2) and (4) present estimates excluding extra time matches. The effect on the magnitude of the parameter estimates is negligible. It was not possible to identify unobserved heterogeneity in the goal-scoring rates. 


\subsection{Sensitivity analysis}

To explore the robustness of our findings, we present a wide range of sensitivity analysis of which the main results are summarized in Table 7. To investigate possible heterogeneity in the effects of a red card on the goal-scoring rate, we introduce interaction effects between a red card and the experience or the age of a player sent off. Such an interaction is also investigated with the line in which the player was deployed (attack, midfield or defense). Interaction effects of both an own and an opponent's red card with the age and experience shown in column (1) suggest that the loss of an older or experienced player does not present a significant advantage. ${ }^{7}$ Column (2) introduces an interaction with the line of play of the player sent off finding no significant effects.

Column (3) investigates whether there is a difference in the shift of the goal-scoring rate resulting from a second yellow red card or straight red card. A foul or misconduct resulting in a straight red card does not have a significantly different impact on the goal-scoring rate either.

We also analyze whether there is a difference in the effect of receiving a red card on the goal-scoring rate of an experienced team. A national team is defined as experienced if a country participated in every World Cup since 1982. Six countries fulfill this criterion-Argentina, Brazil, England, Italy, Germany and Spain—accounting for 12 red cards in the analysis.

The results shown in column (4) indicate that these teams have higher goal-scoring rate after the sending-off of an own player compared to the others. However, the estimated parameter narrowly misses the $10 \%$ significance level. Finally, we investigate whether a different measure of team strength has any effects on the results, as FIFA ranking has been criticized as unreliable. Instead, as before we employ betting odds translated into the expected number of points from a match. As shown in column (5), including the expected number of points as a measure of team strength results in a negligible change in magnitude of the estimates compared to the baseline. The main conclusion holds, as teams with higher expected number of points have higher goal-scoring rate.

\section{Conclusions}

We analyze 320 World Cup matches to investigate both the determinants of the issuing of a red card and the effects of a red card on goal-scoring. We find the rate at which a red card is issued to depend on the goal difference that evolves during a match. Teams who are behind in terms of goals scored are more likely to be confronted with a red card. We also find that the sending-off of a player presents a significant disadvantage for the team that is confronted with a red card. The size of this effect depends on the time the red card was issued.

Our results suggest that the sending-off of a player does not translate into a better performance of the sanctioned team. Hence, our findings are at odds with the "ten do it better" myth. It may be that the loss of a player results in increased motivation of the

\footnotetext{
7 Introducing age-squared does not improve the estimation results.
} 


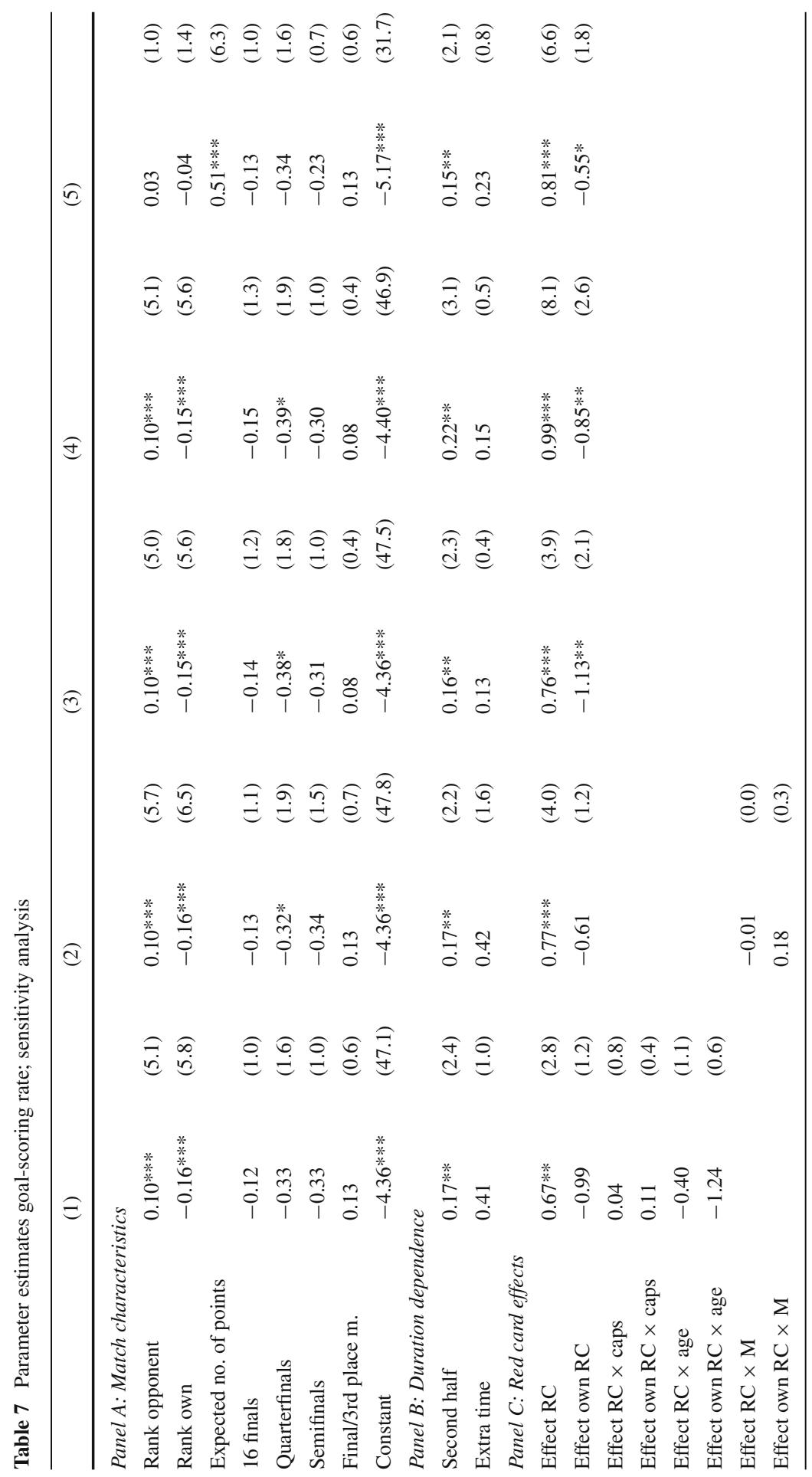




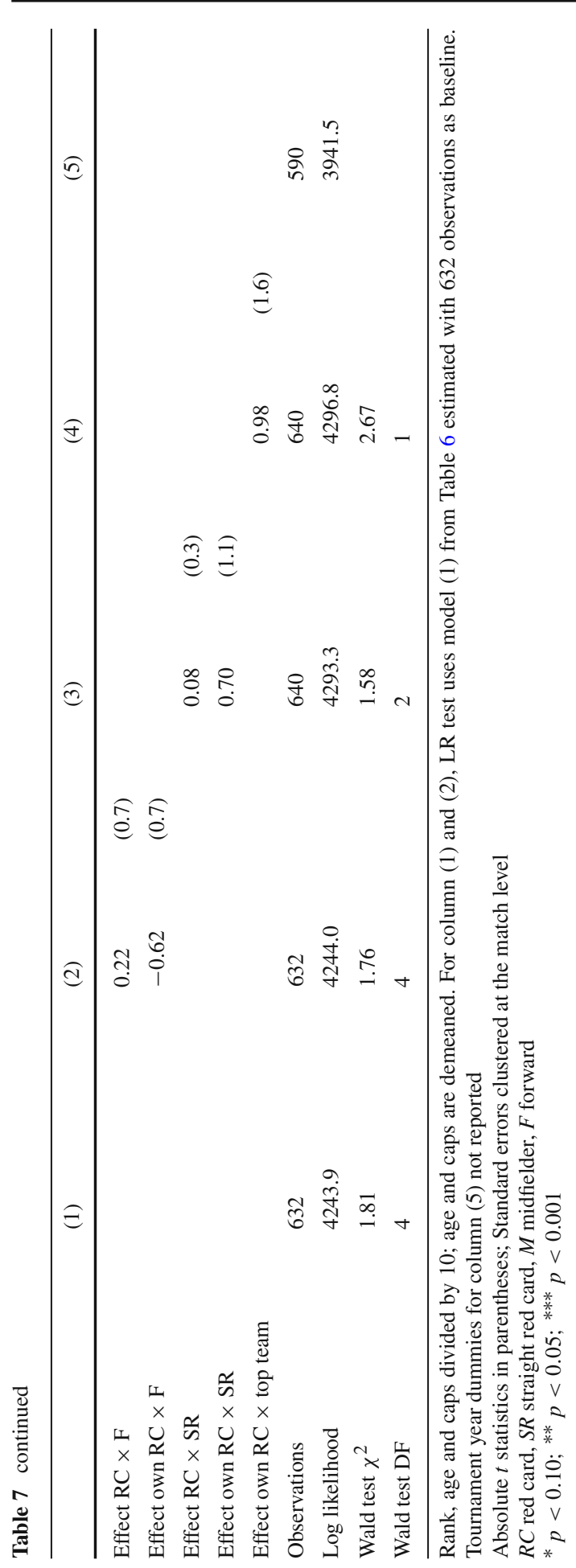


remaining players on the pitch. However, on balance smaller teams do worse. Apparently, the loss of a player in a sports competition presents a significant disadvantage which is be difficult to overcome through increased effort.

Acknowledgements The authors thank two anonymous reviewers for their helpful comments on a previous version of the paper.

Open Access This article is distributed under the terms of the Creative Commons Attribution 4.0 International License (http://creativecommons.org/licenses/by/4.0/), which permits unrestricted use, distribution, and reproduction in any medium, provided you give appropriate credit to the original author(s) and the source, provide a link to the Creative Commons license, and indicate if changes were made.

\section{Appendix: Definition of variables}

Rank own FIFA ranking of the team of which the goal-scoring rate and the red card sanction rate is estimated. The ranking is established before the start of each tournament.

Rank opponent FIFA ranking of the opposing team.

Expected number of points Calculated using pre-match betting odds. These odds are from various Web sites including sports.williamhill.com and www.betexplorer.com.

Stage of a tournament Reference category: group stage.

Goal difference before red card Number of goals scored minus the number of goals conceded at the time the first red card was issued.

Goals scored Number of goals scored.

Same continent referee Dummy variable; takes value of one if the team is from the same continent as the referee. If both teams are from the same continent as the referee, the variable takes value of zero.

Referee rank Based on International Federation of Football History and Statistics annual ranking. We use the ranking of a referee 1 year prior to the tournament (if available).

Age Age of a player at the start of a tournament.

Caps Number of caps of a player at the start of a tournament.

Position of a player Line of a play on the pitch — midfield, attack. Reference category: defense, including goalkeeper. 


\section{References}

Anderson C, Sally D (2013) The numbers game: why everything you know about football is wrong. Penguin Books, London

Bar-Eli M, Tenenbaum G, Geister S (2006) Consequences of players' dismissal in professional soccer: a crisis related analysis of group size effect. J Sport Sci 24(10):1083-1094

Buraimo B, Forrest D, Simmons R (2010) The 12th man? Refereeing bias in English and German soccer. J R Stat Soc Ser A 173(2):431-449. doi:10.1111/j.1467-985X.2009.00604.X

Caliendo M, Radic D (2006) Ten do it better, do they? An empirical analysis of an old football myth. IZA discussion papers 2158. http://EconPapers.repec.org/RePEc:iza:izadps:dp2158

Dawson P, Dobson S, Goddard J, Wilson J (2007) Are football referees really biased and inconsistent? Evidence on the incidence of disciplinary sanction in the English premier league. J R Stat Soc Ser A Stat Soc 170(1):231-250

Heckman J, Singer B (1984) A method for minimizing the impact of distributional assumptions in econometric models for duration data. Econometrica 52(2):271-320

Latané B (1981) The psychology of social impact. Am Psychol 36(4):343-356

Mechtel M, Bäker A, Brändle T, Vetter K (2011) Red cards: not such bad news for penalized guest teams. J Sports Econ 12(6):621-646

Pope BR, Pope NG (2015) Own-nationality bias: evidence from UEFA champions league football referees. Econ Inquiry 53(2):1292-1304. doi:10.1111/ecin.12180

Ridder G, Cramer JS, Hopstaken P (1994) Down to ten: estimating the effect of a red card in soccer. J Am Stat Assoc 89(427):1124-1127

Ringelmann M (1913) Recherches sur les moteurs animes: Travail de homme (research on animate sources of power: The work of man). Annales de l'Institut National Agronomique 2e serie tome XII:1-40

Steiner ID (1972) Group processes and productivity. Academic Press, New York

Titman A, Costain D, Ridall P, Gregory K (2015) Joint modelling of goal and bookings in association football. J R Stat Soc Ser A 178(3):659-683

Van Ours JC, van Tuijl MA (2011) Country-specific goal-scoring in the "dying seconds" of international football matches. Int J Sport Financ 6(2):138-154

Van Ours JC, van Tuijl MA (2016) In-season head-coach dismissals and the performance of professional football teams. Econ Inquiry 54(1):591-604

Vecer J, Kopriva F, Ichiba T (2009) Estimating the effect of the red card in soccer: when to commit an offense in exchange for preventing a goal opportunity. J Quant Anal Sports 5(1):1-20 